\title{
Symmetry-enhanced performance of dynamical decoupling
}

\author{
S. Pasini ${ }^{1, *}$ and G. S. Uhrig ${ }^{2, \dagger}$ \\ ${ }^{1}$ Forschungszentrum Jülich, D-52425 Jülich, Germany \\ ${ }^{2}$ Lehrstuhl für Theoretische Physik I, Technische Universität Dortmund, Otto-Hahn Straße 4, D-44221 Dortmund, Germany
}

(Received 24 August 2011; published 25 October 2011)

\begin{abstract}
We consider a system with general decoherence and a quadratic dynamical decoupling sequence (QDD) for the coherence control of a qubit coupled to a bath of spins. We investigate the influence of the geometry and of the initial conditions of the bath on the performance of the sequence. The overall performance is quantified by a distance norm $d$. It is expected that $d$ scales with $\tau$, the total duration of the sequence, as $\tau^{\min \left\{N_{x}, N_{z}\right\}+1}$, where $N_{x}$ and $N_{z}$ are the number of pulses of the outer and of the inner sequence, respectively. We show both numerically and analytically that the state of the bath can boost the performance of QDD under certain conditions: The scaling of QDD for a given number of pulses can be enhanced by a factor of 2 if the bath is prepared in a highly symmetric state and if the system Hamiltonian is SU(2) invariant.
\end{abstract}

DOI: 10.1103/PhysRevA.84.042336

PACS number(s): 03.67.Pp, 03.65.Yz, 82.56.Jn, 03.67.Lx

\section{INTRODUCTION}

Improvements both in resonance spectroscopy and in quantum information rely on the ability of suppressing unwanted couplings between the system and its environment. Uncontrolled couplings are often the origin of phase accumulation and, in general, of decoherence. Therefore, a faithful manipulation and preservation of quantum states is required.

Dynamical decoupling (DD) is an open-loop control scheme to average out the undesired coupling between the system (qubit) and the environment (bath) by means of stroboscopic pulsing of the qubit. DD was developed by Viola and Lloyd [1] from the original idea of Hahn [2].

In its original formulation the DD makes use of equidistant $\pi$ pulses to average out only a single coupling along one spin direction, usually the $z$ direction, (pure dephasing)—we think, for example, of the Carr, Purcell, Maiboom, and Gill (CPMG) sequence [3,4]. A remarkable advance is the optimal DD discovered by Uhrig [5], whose sequence has the minimum number of pulses for a given order of the suppression of the decoherence. It was shown that Uhrig dynamical decoupling (UDD) can also be used to suppress longitudinal relaxation [6-8]. Recently, other nonequidistant sequences have been proposed [9-11].

The most general case concerns the suppression of dephasing and longitudinal relaxation at the same time. A sequence of pulses having a single level of suppression cannot suppress general dephasing. Sequences with two sorts of pulses have been proposed where concatenated sequences are used, like concatenated dynamical decoupling (CDD) [12] and concatenated Uhrig dynamical decoupling (CUDD) [8], for example.

Recently, West et al. [13] have proposed a near optimal scheme that suppresses arbitrary couplings to order $\tau^{N}(\tau$ is the duration of the total sequence) between the qubit and the bath using $O\left(N^{2}\right)$ pulses. The sequence consists of two levels of nested UDD, therefore the name quadratic UDD (QDD). The validity of UDD can be extended to analytically time-

\footnotetext{
*s.pasini@fz-juelich.de

${ }^{\dagger}$ goetz.uhrig@tu-dortmund.de
}

dependent Hamiltonians [14], which is an important ingredient for the demonstration of QDD.

Wang et al. [15] showed that the effect of QDD can be decomposed in the effects of the inner and the outer sequences. The concept of a mutually orthogonal operation set (MOOS) for nested Uhrig DD was introduced: a set of control operators on the inner level is not affected by a set of control operators on the outer level if both sets come from a MOOS. Higherorder protection of a MOOS can be achieved if even-order UDD sequences on different levels are nested. Thus, the results in Ref. [15] demonstrate the validity of QDD with an evenorder UDD sequence on the inner level. If the inner level has an odd order, the symmetry group generated by MOOS is broken and the scheme based on nested UDD cannot be applied anymore. It appears that this problem has been solved by Jiang and Imambekov [16], who have provided a proof of the validity of nested UDD (NUDD) sequences that relies on a mapping between NUDD and a discrete quantum walk in $2 m$ dimensional space. The case of QDD corresponds to $m=1$. At last, an alternative proof of the validity of QDD and a numerical investigation of the scaling of the errors along specific spin directions for QDD has been presented in Ref. [17] and in Ref. [18], respectively.

In this paper we want to draw the attention to the effects of the state of the bath on the performance of the sequence. The fact that the specifics of the bath can limit the performance of a sequence is already known $[19,20]$. It was tested experimentally that UDD can outperform CPMG if the environment is characterized by a hard cutoff $[9,21]$ while for soft cutoffs equidistant sequences perform either better or the same [22]. Otherwise UDD seems to perform very well for electron spins in irradiated malonic acid crystals [21] as well as for applications of magnetic resonance imaging [23].

So far we have always viewed the environment as an unavoidable restraint on the prolongation of the coherence of a spin (qubit). Hence, one has to eliminate or at least to reduce the coupling between environment and system because the coupling between environment and system transfers disorder from the environment to the system. But does the environment's disorder always act against coherence in the system? 
TABLE I. Scaling of QDD with the duration of the sequence $\tau$ classified according to the degree of symmetry of the Hamiltonian and of the initial state of the bath. The notation $N_{\min }$ refers to the minimum number of pulses of the inner and the outer sequence.

\begin{tabular}{lcc}
\hline \hline$\rho_{\mathrm{B}} \backslash H$ & Low & High \\
\hline Low & $\tau^{N_{\min }+1}$ & $\tau^{N_{\min }+1}$ \\
High & $\tau^{\chi}$ & $\tau^{2\left(N_{\min }+1\right)}$ \\
\hline \hline
\end{tabular}

Here we show that the performance of a given sequence can be enhanced if the system Hamiltonian $H$ is SU(2) invariant and if the initial state of the bath (i.e., its density matrix $\rho_{\mathrm{B}}$ ) is completely disordered: $\rho_{\mathrm{B}} \propto \mathbb{1}$. We call such a state an infinite-temperature state. We simulate the effect of a QDD sequence on a bath of spins both for a completely anisotropic as well as for an isotropic central Heisenberg spin model. For both cases we analyze the scaling of QDD when the bath is prepared either in a product state or in an infinite-temperature state. Four cases are studied as summarized in Table I. The cases where the bath state is characterized by a low degree of symmetry, independent of the degree of symmetry of $H$, provide the lower bounds for the scaling of QDD: For short times, QDD scales always as $\tau^{N_{\min }+1}$, where $N_{\min }$ is the smallest number of pulses, either of the inner or of the outer sequence $[17,18]$. In the off-diagonal case in Table I, where $H$ is of low symmetry and the bath state of high symmetry, the scaling exponent $\chi$ depends on the number of pulses. Otherwise, the scaling of QDD is enhanced to the power $\tau^{2\left(N_{\min }+1\right)}$ if $H$ and $\rho_{\mathrm{B}}$ are highly symmetric.

The paper is set up as follows: in Sec. II and in the Sec. III the numerical results for the low- and high-symmetry cases are presented. In Sec. IV we provide the analytical argument for the appearance of the factor 2 in the high-symmetry case. In Sec. V we study the off-diagonal cases with mixed symmetry. At last we draw our conclusions in Sec. VI.

\section{CASE 1: LOW SYMMETRY}

We start from the case where both the system Hamiltonian as well as the initial state of the bath have a low degree of symmetry. We consider a central spin model [24-28] characterized by a completely anisotropic Hamiltonian of the form

$$
\begin{gathered}
H=H_{\mathrm{B}}+H_{\mathrm{qB}} \\
=\sum_{i=1}^{M} \sum_{j>i}^{M} \vec{\sigma}^{(i)} \hat{J}_{0}^{i j} \vec{\sigma}^{(j)}+\sum_{i=1}^{M} \vec{\sigma}^{(0)} \hat{J}_{1}^{i} \vec{\sigma}^{(i)},
\end{gathered}
$$

where all nine entries of the $3 \times 3 \hat{J}_{0}^{i j}$ and $\hat{J}_{1}^{i}$ matrices are random numbers drawn from the interval $[-1,1]$ (see Fig. 1). The system does not show any symmetry. The entries for the matrices $\hat{J_{0}^{i j}}$ and $\hat{J_{1}^{i}}$ are fixed randomly at the beginning of the simulation and they remain the same for all the numerical results we present in this article. The spin labeled with zero represents the qubit while $M$ defines the number of spins in the bath. The scaling appears to be essentially independent [29] of $M$; we considered for our simulation $M=8$. Calculations

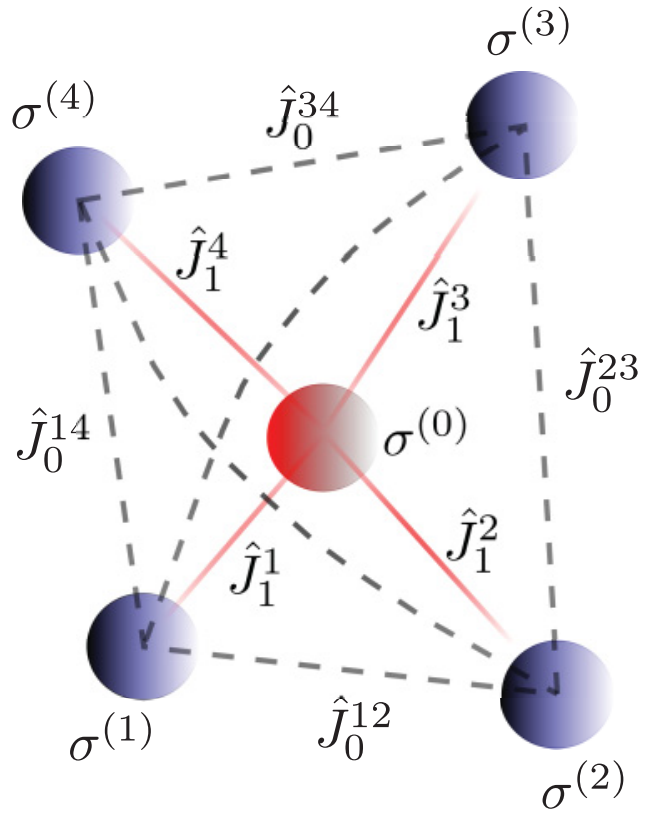

FIG. 1. (Color online) Central spin model described by Eq. (1) with $M=4$. The solid lines represent the coupling between the central spin (qubit) and the spins of the bath, while the dashed lines represent the couplings between the spins of the bath.

for $M=3$ yield the same results as far as the exponents are concerned.

The QDD sequence is made of an outer sequence of $\pi$ pulses about $\sigma_{x}$ and of an inner sequence of $\pi$ pulses about $\sigma_{z}$. The number of pulses for each sequence is $N_{x}$ and $N_{z}$, respectively, and the total number of pulses of the sequence is $N_{x}+N_{z}+N_{x} N_{z}$. The switching instants are given by

$$
\begin{gathered}
t_{j}^{x}=\tau \sin ^{2}\left[\frac{j \pi}{2\left(N_{x}+1\right)}\right], \\
t_{k, j}^{z}=t_{j}^{x}+\left(t_{j+1}^{x}-t_{j}^{x}\right) \sin ^{2}\left[\frac{k \pi}{2\left(N_{z}+1\right)}\right],
\end{gathered}
$$

for $j=\left\{1, \ldots, N_{x}\right\}$ and $k=\left\{1, \ldots, N_{z}\right\}$. We use the notation $t_{N_{x}+1}^{x}=\tau$.

We start with an initial density matrix of the total system of the form

$$
\rho_{0}^{(\gamma)}=|\gamma\rangle\langle\gamma| \otimes \rho_{\mathrm{B}},
$$

with $\gamma=\{x, y, z\}$. The first factor in the tensor product refers to the Hilbert space of the qubit, the second to the Hilbert space of the bath. Furthermore, we introduce the notation

$$
\rho_{0}^{(\gamma)}:=\rho_{\mathrm{S}}^{(\gamma)} \otimes \rho_{\mathrm{B}}
$$

For the low-symmetry case we assume that the bath is initially in a pure product state:

$$
\left|\psi_{\mathrm{B}}\right\rangle=\bigotimes_{i=1}^{M}\left|\gamma_{i}\right\rangle,
$$

so that $\rho_{\mathrm{B}}=\left|\psi_{\mathrm{B}}\right\rangle\left\langle\psi_{\mathrm{B}}\right|$. For the high-symmetry case we choose

$$
\rho_{\mathrm{B}}=\mathbb{1}_{\mathrm{B}} / D,
$$

where $D$ is the dimension of the Hilbert space of the bath so that $\operatorname{Tr}_{\mathrm{B}} \rho_{\mathrm{B}}=1$. 
The overall performance of the sequence is given by the norm distance [30]:

$$
\begin{aligned}
& d^{2}:=\frac{1}{3} \sum_{\gamma \in\{x, y, z\}} d_{\gamma}^{2}, \\
& d_{\gamma}^{2}:=\operatorname{Tr}_{\mathrm{q}}\left[\Delta^{(\gamma)}(\tau)\right]^{2},
\end{aligned}
$$

with

$$
\Delta^{(\gamma)}(\tau):=\operatorname{Tr}_{\mathrm{B}}\left[U_{\mathrm{B}} \hat{P} \rho_{0}^{(\gamma)} \hat{P}^{\dagger} U_{\mathrm{B}}^{\dagger}-U(\tau, 0) \rho_{0}^{(\gamma)} U(\tau, 0)^{\dagger}\right] .
$$

The norm distance measures the distance of the real evolution to the ideal one. The operator $\hat{P}$ is defined by

$$
\hat{P}:=\underbrace{\sigma_{z}^{N_{z}} \sigma_{x} \sigma_{z}^{N_{z}} \sigma_{x} \cdots \sigma_{z}^{N_{z}}}_{N_{x}+1} .
$$

It incorporates the effects of the pulses. The operator $U(\tau, 0)$ represents the evolution operator of the system (1):

$$
U(\tau, 0)=\mathcal{T}\left\{e^{-i \int_{0}^{\tau} H(t) d t}\right\},
$$

where $\mathcal{T}$ stands for the time-ordering and

$$
U_{\mathrm{B}}:=\mathbb{1} \otimes e^{-i \tau H_{\mathrm{B}}}
$$

is the dynamics of the isolated bath. During the application of the sequence, the sign in front of the coupling terms between the qubit and the bath perpendicular to the pulse direction changes every time a $\pi$ pulse is applied because $\sigma_{i} \sigma_{j}=-\sigma_{j} \sigma_{i}$ for $i \neq j$. Thus, in the toggling frame, the system Hamiltonian (1) can be written as a time-dependent Hamiltonian

$$
H(t)=H_{\mathrm{B}}+\sum_{i=1}^{M} \sum_{j, k=1}^{3} f_{j}(t) \sigma_{j}^{(0)}\left(J_{1}^{i}\right)_{j k} \sigma_{k}^{(i)},
$$

where the switching functions $f_{j}(t)$ are a piecewise constant functions with values \pm 1 . By $\sigma_{k}^{(i)}$ we refer to the $k$ component of the vector $\vec{\sigma}^{(i)}$. Analogously, we refer by $\left(J_{1}^{i}\right)_{j k}$ to the $(j, k)$ element of the matrix $\hat{J}_{1}^{i}$.

Our simulations show that, for $N_{x} \neq 0, N_{z} \neq 0$, and

$$
\begin{aligned}
& \text { for } N_{x}=N_{z}=N \rightarrow d \propto \tau^{N+1}+O\left(\tau^{N+2}\right), \\
& \quad \text { for } N_{x}>N_{z} \rightarrow d \propto \tau^{N_{z}+1}+O\left(\tau^{N_{z}+2}\right), \\
& \quad \text { for } N_{x}<N_{z} \rightarrow d \propto \tau^{N_{x}+1}+O\left(\tau^{N_{x}+2}\right),
\end{aligned}
$$

as shown in Table II and in Fig. 2(a). The data agree with the results of Ref. [18] for the overall error. For either $N_{x}=0$ or $N_{z}=0$ (UDD sequence) we find that $d$ scales as $\tau$, as expected for a Hamiltonian with general decoherence. On the other hand, if $N_{z}=0$ for example and $H_{\mathrm{qB}}$ is a pure dephasing Hamiltonian (i.e., no couplings with $\sigma_{x}^{(0)}$ or with $\sigma_{y}^{(0)}$ occur in the Hamiltonian), the norm distance scales as $d_{\mathrm{UDD}} \propto \tau^{N_{x}+1}$.

Here we present the results of a given random configuration of the entries of $\hat{J}_{0}^{i j}$ and $\hat{J}_{1}^{i}$. We also checked that the scaling is the same for other random configurations.

Different choices of the initial bath state $\rho_{\mathrm{B}}$ of the form (5) (i.e., varying the $\left|\gamma_{i}\right\rangle$ ) can affect the scaling of $d_{x}, d_{y}$, and $d_{z}$, but not the overall scaling of $d$ : If $d_{\gamma} \propto \tau^{A_{\gamma}}$ then the leading order of the norm distance scales as $d \propto \tau^{\min _{\gamma}\left\{A_{\gamma}\right\}}$. Hence the scaling exponent reads $\zeta=\min _{\gamma}\left\{A_{\gamma}\right\}$. This is what the analytic arguments require for QDD [15-17]. Hence the analytic bounds on the exponents are sharp for the low-symmetry case.
TABLE II. Low-symmetry case: Scaling exponent $\zeta$ of the norm distance $d(\tau)$ with $\tau$, the total duration of the sequence. The reported numbers are determined from the slope of the curve $d$ vs $\tau$ in a double logarithmic plot.

\begin{tabular}{lccccccc}
\hline \hline$N_{z} \backslash N_{x}$ & 0 & 1 & 2 & 3 & 4 & 5 & 6 \\
\hline 0 & 1.00 & 1.00 & 1.00 & 1.00 & 1.00 & 1.00 & 1.00 \\
1 & 1.00 & 2.01 & 2.00 & 2.01 & 2.01 & 2.01 & 2.00 \\
2 & 1.00 & 2.01 & 2.97 & 3.01 & 3.01 & 3.01 & 3.02 \\
3 & 1.00 & 2.00 & 2.99 & 4.01 & 4.07 & 4.09 & 4.09 \\
4 & 1.00 & 2.00 & 3.00 & 4.01 & 4.99 & 4.98 & 5.05 \\
5 & 1.00 & 1.95 & 3.02 & 3.95 & 5.01 & 5.98 & 6.05 \\
6 & 1.00 & 1.98 & 3.01 & 3.94 & 5.00 & 5.84 & 6.95 \\
\hline \hline
\end{tabular}

\section{CASE 2: HIGH SYMMETRY}

We consider an SU(2) invariant isotropic central spin model with Heisenberg couplings. We choose a Hamiltonian of the form of Eq. (1) with $\hat{J}_{0}^{i j}=\alpha \lambda j_{0}^{i j} \mathbb{1}$ and $\hat{J}_{1}^{i}=\lambda j_{1}^{i} \mathbb{1}$, where $\alpha$ and $\lambda$ are two generic constants while $j_{0}^{i j}$ and $j_{1}^{i}$ are random numbers between -1 and 1 .

If the bath at $t=0$ is described by the following density matrix:

$$
\rho_{\mathrm{B}} \propto \mathbb{1}_{\mathrm{B}},
$$

the suppression of the decoherence is enhanced by a factor 2 . The simulation for QDD yields the scaling exponents reported in Table III. We deduce the following rules: for $N_{x} \neq 0, N_{z} \neq 0$ and

$$
\begin{gathered}
N_{x}=N_{z}=N \rightarrow d \propto \tau^{2(N+1)}+O\left(\tau^{2 N+3}\right), \\
N_{x}>N_{z} \rightarrow d \propto \tau^{2\left(N_{z}+1\right)}+O\left(\tau^{2 N_{z}+3}\right), \\
N_{x}<N_{z} \rightarrow d \propto \tau^{2\left(N_{x}+1\right)}+O\left(\tau^{2 N_{x}+3}\right) .
\end{gathered}
$$

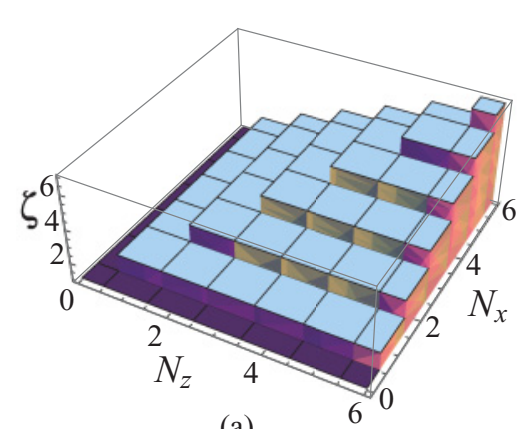

(a)

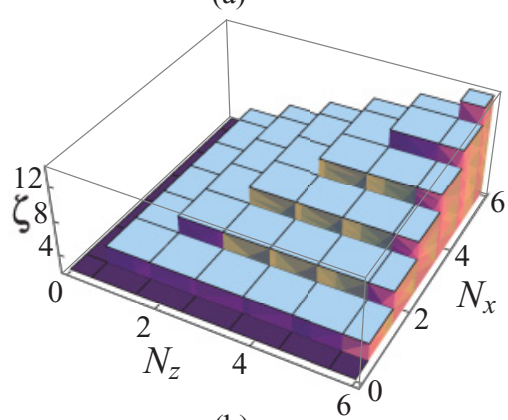

(b)

FIG. 2. (Color online) The data of Tables II and III are graphically represented in panel (a) and in panel (b), respectively. The numbers are rounded to their first digit. Here we have introduced the notation $d \propto \tau^{\zeta}$, where $\zeta$ stands for the scaling exponents. 
TABLE III. High-symmetry case: Scaling exponent $\zeta$ of the norm distance with $\tau$ for an isotropic Hamiltonian with general decoherence and for an initial density matrix such as in Eq. (14). The scaling exponents are derived from a fit of the numerical curves for $d$ vs $\tau$ in a double logarithmic plot.

\begin{tabular}{lccccccc}
\hline \hline$N_{z} \backslash N_{x}$ & 0 & 1 & 2 & 3 & 4 & 5 & 6 \\
\hline 0 & 1.99 & 2.00 & 2.00 & 2.00 & 2.00 & 2.00 & 2.00 \\
1 & 2.00 & 3.99 & 3.95 & 4.00 & 3.98 & 4.00 & 4.00 \\
2 & 2.00 & 4.00 & 5.99 & 6.13 & 6.00 & 5.99 & 5.99 \\
3 & 2.00 & 3.99 & 5.99 & 7.98 & 7.97 & 8.01 & 8.01 \\
4 & 2.00 & 4.00 & 5.99 & 7.99 & 9.97 & 9.92 & 9.94 \\
5 & 2.00 & 4.00 & 5.99 & 7.99 & 9.97 & 11.93 & 11.94 \\
6 & 2.00 & 4.00 & 6.00 & 7.97 & 9.95 & 12.01 & 13.95 \\
\hline \hline
\end{tabular}

If $N_{x}=0$ or $N_{z}=0$ the norm distance scales as $\tau^{2}$. A graphical representation of the data is provided in Fig. 2(b).

The state of Eq. (14) is a completely disordered state where no particular spin direction or state is singled out. Such a state can be referred to as an "infinite-temperature" state. This is not unusual in NMR experiments where already at room temperature one finds $\hbar \omega_{\mathrm{L}} /\left(k_{\mathrm{B}} T\right) \approx 10^{-5}$, where $\omega_{\mathrm{L}}$ is the Larmor frequency of a spin and $k_{\mathrm{B}}$ is the Boltzmann constant. This means that the thermal energy exceeds all internal energy scales by many orders of magnitude.

Note that for $\rho_{\mathrm{B}} \propto \mathbb{1}_{\mathrm{B}}$ the norm distance (7) coincides with the partial Frobenius norm distance $[29,30]$.

\section{SU(2) INVARIANCE}

The appearance of the factor 2 can be explained in terms of the different parity of $H_{\mathrm{B}}$ and $H_{\mathrm{qB}}$ under spin rotations. We write the Hamiltonian (1) in the form

$$
H=\mathbb{1}_{\mathrm{S}} \otimes A_{0}+\sum_{\mu \in\{x, y, z\}} \sigma_{\mu} \otimes A_{\mu} .
$$

In Eq. (16) the operators $A_{0}$ and $A_{\mu}$ act only on the bath while $\mathbb{1}_{\mathrm{S}}$ and $\sigma_{\mu}$ act only on the qubit. Since the identity operator and the Pauli matrices form a complete basis for all system operators the evolution operator can be expanded according to

$$
U(\tau, 0)=\mathbb{1}_{\mathrm{S}} \otimes B_{0}(\tau)+\sum_{\mu=x, y, z} \sigma_{\mu} \otimes B_{\mu}(\tau),
$$

where $B_{0}(\tau)$ and $B_{\mu}(\tau)$ are nontrivial functions of the operators $A_{0}$ and $A_{\mu}$ and of the switching functions $f_{\mu}(t)$, see Eq. (A2) in the appendix and Ref. [31]. For the sake of simplicity we will omit the time dependence of the operators $B_{0}$ and $B_{\mu}$ from now on. From the unitarity of $U(\tau, 0)$ we conclude

and

$$
\mathbb{1}_{\mathrm{B}}=B_{0} B_{0}^{\dagger}+\sum_{\mu \in\{x, y, z\}} B_{\mu} B_{\mu}^{\dagger}
$$

$$
0=i \sum_{\mu, \nu \in\{x, y, z\}} \epsilon_{\mu \nu \kappa} B_{\mu} B_{\nu}^{\dagger}+\left(B_{0} B_{\kappa}^{\dagger}+\text { H.c. }\right)
$$

for fixed $\kappa \in\{x, y, z\}$ and with $\epsilon_{\mu \nu \kappa}$ being the Levi-Civita symbol. In (18b) we omitted the nonsingular factor proportional to Pauli matrices because the vanishing must be ensured by the bath operators. In the Heisenberg picture the density matrix $\rho_{0}^{(\gamma)}(3)$ evolves according to

$$
\begin{aligned}
& \rho_{0}^{(\gamma)}(\tau)=U(\tau, 0) \rho_{0}^{(\gamma)} U(\tau, 0)^{\dagger} \\
& =U(\tau, 0)\left(\rho_{\mathrm{S}}^{(\gamma)} \otimes \rho_{\mathrm{B}}\right) U(\tau, 0)^{\dagger} .
\end{aligned}
$$

We trace out the bath and use the unitarity of $U(\tau, 0)(18)$ to obtain

$$
\operatorname{Tr}_{\mathrm{B}} \rho_{0}^{(\gamma)}(\tau)=T_{1}^{(\gamma)}+T_{2}^{(\gamma)}+T_{3}^{(\gamma)}+T_{4}^{(\gamma)},
$$

with

$$
\begin{aligned}
T_{1}^{(\gamma)}:=\rho_{\mathrm{S}}^{(\gamma)} \operatorname{Tr}_{\mathrm{B}} \rho_{\mathrm{B}}+\sum_{\mu \in\{x, y, z\}}\left(c_{\mu, \mu}^{(\gamma)}-\rho_{\mathrm{S}}^{(\gamma)}\right) b_{\mu, \mu}, \\
T_{2}^{(\gamma)}:=\sum_{\mu, \nu \in\{x, y, z\}, \mu \neq \nu} c_{\mu, \nu}^{(\gamma)} b_{\mu, \nu}, \\
T_{3}^{(\gamma)}:=\sum_{\mu \in\{x, y, z\}} d_{\mu}^{(\gamma)} b_{\mu}, \\
T_{4}^{(\gamma)}:=-i \sum_{\mu, \nu, \kappa \in\{x, y, z\}} \epsilon_{\mu \nu \kappa} \rho_{\mathrm{S}}^{(\gamma)} \sigma_{\kappa} b_{v, \mu} .
\end{aligned}
$$

The coefficients

$$
\begin{aligned}
b_{\mu, v} & :=\operatorname{Tr}_{\mathrm{B}}\left[B_{\mu} \rho_{\mathrm{B}} B_{v}^{\dagger}\right], \\
b_{\mu} & :=\operatorname{Tr}_{\mathrm{B}}\left[B_{0} \rho_{\mathrm{B}} B_{\mu}^{\dagger}\right],
\end{aligned}
$$

depend only on the bath operators while $c_{\mu, \nu}^{(\gamma)}:=\sigma_{\mu} \rho_{\mathrm{S}}^{(\gamma)} \sigma_{\nu}$ and $d_{\mu}^{(\gamma)}:=\sigma_{\mu} \rho_{\mathrm{S}}^{(\gamma)}-\rho_{\mathrm{S}}^{(\gamma)} \sigma_{\mu}$ are functions of the qubit operators only. Note that, for a pure dephasing model [31], the terms $T_{2}^{(\gamma)}$ and $T_{4}^{(\gamma)}$ do not appear.

We consider a global operator $\hat{P}_{v}$ that rotates all the spins of our system around the $v=x, y$, or $z$ axis by the angle $\pi$. Here we are interested in the $\mathrm{SU}(2)$-invariant Hamiltonian such as the one discussed in Sec. III. Then we have

$$
\begin{gathered}
\hat{P}_{v} B_{0} \hat{P}_{v}^{\dagger}=B_{0}, \quad \hat{P}_{v} B_{v} \hat{P}_{v}^{\dagger}=B_{v}, \\
\hat{P}_{v} B_{\mu} \hat{P}_{v}^{\dagger}=-B_{\mu} \quad \text { for } v \neq \mu
\end{gathered}
$$

and, therefore,

$$
\begin{gathered}
b_{\mu}=\operatorname{Tr}_{\mathrm{B}}\left[B_{0} \rho_{\mathrm{B}} B_{\mu}^{\dagger}\right] \\
=\operatorname{Tr}_{\mathrm{B}}\left[\hat{P}_{\nu} B_{0} \hat{P}_{\nu}^{\dagger} \hat{P}_{\nu} \rho_{\mathrm{B}} \hat{P}_{\nu}^{\dagger} \hat{P}_{\nu} B_{\mu}^{\dagger} \hat{P}_{\nu}^{\dagger}\right] \\
=-\operatorname{Tr}_{\mathrm{B}}\left[B_{0} \hat{P}_{\nu} \rho_{\mathrm{B}} \hat{P}_{v}^{\dagger} B_{\mu}^{\dagger}\right]
\end{gathered}
$$

for $\mu \neq v$. Thus, if $\rho_{\mathrm{B}}$ is invariant under rotation $\hat{P}_{v}$, which is the case for $\rho_{\mathrm{B}} \propto \mathbb{1}_{\mathrm{B}}$, we can conclude from (25c) that $b_{\mu}=0$ for $\mu \in\{x, y, z\}$. In fact, the analogous argument also implies $b_{\mu, v}=0$ for $\mu \neq v$, although we will not use this fact here. The condition $\mu \neq v$ is needed to ensure that we can flip the sign of the two factors $B_{\mu}$ and $B_{v}^{\dagger}$ separately.

If the coefficients $b_{\mu}$ vanish only the terms proportional to $b_{\mu, \nu}$ remain in Eq. (21). We know from the analytic properties of the QDD sequence that the operators $B_{\mu}$ with $\mu \in\{x, y, z\}$ all scale at least with $\tau^{N_{\min }+1}$ where $N_{\min }:=\min \left(N_{x}, N_{z}\right)$ [15-17], which is supported by numerical results in Ref. [18] and in the present work. Hence the coefficients $b_{\mu}$ scale with $\tau^{N_{\min }+1}$ and the coefficients $b_{\mu, v}$ with $\tau^{2 N_{\min }+2}$. Hence, the vanishing of the $b_{\mu}$ terms in Eq. (21) automatically reduce 
TABLE IV. Mixed cases: In Table IV(a) (left) the Hamiltonian is SU(2) invariant while the bath is initially prepared in a product state. In Table IV(b) (right) the Hamiltonian is asymmetric of the form in Eq. (1), the entries of $\hat{J}_{0}^{i j}$ and $\hat{J}_{1}^{i}$ are all randomly chosen, and $\rho_{\mathrm{B}} \propto \mathbb{1}_{\mathrm{B}}$.

\begin{tabular}{lccccccccccccccc}
\hline \hline$N_{z} \backslash N_{x}$ & 0 & 1 & 2 & 3 & 4 & 5 & 6 & $N_{z} \backslash N_{x}$ & 0 & 1 & 2 & 3 & 4 & 5 \\
\hline 0 & 0.99 & 1.00 & 1.00 & 1.00 & 1.00 & 1.00 & 1.00 & 0 & 1.98 & 2.00 & 2.00 & 2.00 & 2.00 & 2.00 & 2.00 \\
1 & 1.00 & 2.00 & 2.00 & 2.00 & 2.00 & 2.00 & 2.00 & 1 & 2.00 & 2.00 & 2.00 & 2.00 & 2.00 & 2.00 & 2.00 \\
2 & 1.00 & 1.99 & 3.01 & 3.00 & 3.00 & 3.00 & 3.00 & 2 & 2.00 & 3.99 & 6.00 & 5.75 & 5.99 & 5.99 & 5.99 \\
3 & 1.00 & 2.00 & 3.00 & 4.00 & 4.00 & 4.00 & 4.00 & 3 & 2.00 & 4.00 & 5.80 & 4.00 & 4.00 & 4.00 & 4.00 \\
4 & 1.00 & 2.01 & 3.00 & 4.02 & 5.00 & 5.00 & 5.01 & 4 & 2.00 & 4.00 & 6.00 & 7.96 & 9.96 & 10.27 & 10.30 \\
5 & 1.00 & 2.00 & 3.00 & 4.00 & 5.02 & 6.00 & 6.00 & 5 & 2.00 & 3.97 & 5.97 & 7.97 & 9.85 & 6.00 & 6.00 \\
6 & 1.00 & 2.00 & 3.00 & 3.98 & 5.00 & 6.00 & 6.99 & 6 & 2.00 & 4.00 & 5.99 & 7.99 & 9.95 & 11.93 & 13.92 \\
\hline \hline
\end{tabular}

the decoherence by doubling the exponent in the scaling $d \propto$ $\tau^{N_{\min }+1} \rightarrow \tau^{2 N_{\min }+2}$ with the total duration $\tau$ of the sequence.

Note that, for a model of pure dephasing [e.g., only $\sigma_{z}^{(0)}$ appears in (1b)], we do not need the symmetry with respect to two operators $\hat{P}_{\mu}$. It is sufficient to have either $\hat{P}_{x}$ or $\hat{P}_{y}$ which invert $\sigma_{z}$ so that we can conclude that $b_{z}$ vanishes in order to know that the scaling exponent doubles. This was already seen in the numerical data presented and analyzed in Ref. [29].

\section{CASE 3: MIXED SYMMETRY}

Here we analyze the off-diagonal cases of Table I. They are characterized either by an SU(2)-invariant Hamiltonian and a low-symmetry bath state or by a low-symmetry Hamiltonian with a high-symmetry initial bath state $\propto \mathbb{1}_{\mathrm{B}}$. Because the Hamiltonian and the density matrix have a different degree of symmetry the analytical argument of Eq. (25a) for $b_{\mu}$ does not hold anymore.

The numerically found scaling exponents are reported in Tables IV and depicted in Fig. 3. The coupling constants used to derive the scaling exponents of this table are the same as those used for the simulation of the SU(2)-invariant Hamiltonian or the ones used for the asymmetric Hamiltonians used in Secs. II and III.

If the Hamiltonian is SU(2) symmetric and the bath is initially prepared in a product state the scaling exponents look the same as those of Table II. If the Hamiltonian is asymmetric and $\rho_{\mathrm{B}} \propto \mathbb{1}_{\mathrm{B}}$ we find for $N_{x}=N_{z}=N$ that the scaling exponent is $N+1$ for $N$ odd and $2(N+1)$ for $N$ even. For $N_{x}>N_{z}$ the exponent is either $N_{z}+1$ if $N_{z}$ is odd or $2\left(N_{z}+1\right)$ if $N_{z}$ is even while for $N_{x}<N_{z}$ we find that $d$

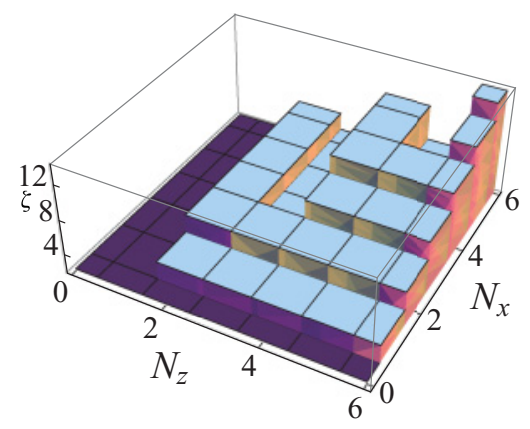

FIG. 3. (Color online) The data of Table IV(b) is graphically represented. The numbers are rounded to their first digit. The notation $\zeta$ refers to the scaling exponents of the norm distance $d \propto \tau^{\zeta}$. scales as $2\left(N_{x}+1\right)$. We cannot provide any explanations for this alternating behavior of the scaling exponents and for the reason why, for $N_{x}>N_{z}$, it depends only on the number of pulses of the inner sequence. This is still an open question for future investigation.

Furthermore, it is worth mentioning that peculiar choices of the couplings imply nongeneric behavior. If the couplings $j_{1}^{i}$ is the same for all $i$ the QDD sequence behaves like a UDD sequence with $N_{z}$ pulses for $N_{x}$ odd, as if the qubit were subject only to pure dephasing: Either $A_{x} \neq 0$ or $A_{y} \neq 0$ in Eq. (16). It is not clear why the QDD behaves like a UDD sequence in this case. A possible explanation is that the product state we take as initial state of the bath is not the most general one. It is not entangled. In the appendix we analyze the first three cumulants of the evolution operator for the case $N_{x}=1$. We find that they contain only one qubit operator, either $\sigma_{x}$ or $\sigma_{y}$, and that the dephasing term proportional to $\sigma_{z}$ is always zero. For $N_{x}$ even we recover the results of Table II.

\section{CONCLUSIONS}

We investigated the influence of the state of the bath in suppressing general decoherence by means of a QDD sequence. The performance of the sequence is measured by the norm distance $d$ which is essentially the norm of the remaining decoherence. Thus the performance is quantified by the scaling of $d$ with $\tau$, the total duration of the sequence. Recent papers [15-18] proved the properties of QDD and clarified the dependence of the scaling on the number of pulses $N_{x}$ and $N_{z}$ : The overall scaling of QDD is given by $\zeta:=\min \left\{N_{x}, N_{z}\right\}+1$ independent of the details of the environment (i.e., $d \propto \tau^{\zeta}$ ). In this sense QDD is a universal sequence for general decoherence such as UDD is a universal sequence for pure dephasing.

In the present work, we have shown that the actual performance of QDD can be even better than expected on the basis of the general mathematical arguments. This improvement occurs if the Hamiltonian and the bath state are highly symmetric; for instance, if the Hamiltonian is spin isotropic and the bath is prepared initially in a completely disordered state. Then we found both numerically and analytically that the exponent of the scaling with $\tau$ acquires an additional factor of $2: d \propto \tau^{2 \zeta}$. The same was already observed for the UDD sequence applied to pure dephasing in Ref. [29].

We emphasize that this result is by no means at odds with the proofs of universality [15-17]. The general proofs refer 
to the worst case for decoherence. They determine whether a certain operator (Pauli matrix) for the qubit occurs or not irrespective of the bath operator with which it is multiplied. The underlying idea is that, for any nonvanishing bath operator there is a bath state such that the qubit state is influenced in a nontrivial way. Hence decoherence occurs.

But for certain choices of the bath state even a nonvanishing bath operator may have a vanishing effect on the quantum bit if its partial bath trace vanishes. Then no decoherence is induced by this particular term. This is the effect which enhances the performance of the QDD sequence for highly symmetric situations. We summarize that $\min \left\{N_{x}, N_{z}\right\}+1$ is a lower bound for $\zeta$.

We stress that the phenomenon found is relevant for realistic situations. Complete or partial spin symmetry in the Hamiltonian is a standard feature. A completely disordered bath state is also an excellent starting point in the description of baths of nuclear spins. Their mutual interaction is so small in energy that even room temperature suffices to disorder the nuclear spins completely.

In general, we conclude that the more asymmetric are the bath Hamiltonian, its coupling to the qubit, and in particular its initial state, the lower the exponent $\zeta$ is of the leading nonvanishing power in the total duration $\tau$ of the sequence inducing decoherence. The same is true of UDD sequences for pure dephasing. So far, we focused on spin baths which allow for completely disordered, infinite temperature states. It is an interesting question for future research whether a similar phenomenon can occur in other baths such as bosonic ones.

Experimental research is also called for. To our knowledge, there exist studies on the influence of the initial state, see for instance Ref. [32], but they focus on the initial state of the system. Discussions of the influence of the initial state of the bath, which was our focus here, are scarce [33]. Moreover, it must be distinguished between studies of iterated cycles of sequences with exponential decay rates [22] and studies of a single sequence displaying decoherence with a particular power law [5-7,13,15-17,28].

Of course, it is difficult to measure the exponents directly. But we suggest to demonstrate experimentally that the performance of a QDD or a UDD sequence is lowered if the symmetry of either the Hamiltonian or of the initial bath state is lowered. This would already be smoking-gun evidence for the essence of the present theoretical finding.

\section{ACKNOWLEDGMENTS}

We would like to thank Gregory Quiroz and Daniel A. Lidar for helpful discussions. The financial support by the grant UH 90/5-1 of the DFG is gratefully acknowledged.

\section{APPENDIX: MAGNUS EXPANSION OF EVOLUTION OPERATOR}

In general, it is complicated to find a universal argument that explains the scaling of QDD if the symmetry of the system is not well defined. The reason why some exponents deviate from the analytically predicted value depends on the possible vanishing of some terms in the Magnus expansion [34,35] of the evolution operator. The form of such terms strongly depends on the form of the Hamiltonian and of the symmetry of the bath state.

If no general conclusions can be drawn on the parity of these terms and of the density matrix under a rotation $\hat{P}_{\mu}$, one way to proceed is to calculate the cumulants of the expansion explicitly and to analyze which terms determine the power law of $d$ with $\tau$. Here we provide an analysis of the first cumulants of the Magnus expansion for $N_{x}$ odd for the data of Table IV(a).

For a generic instant $t \in[0, \tau]$ we write the Hamiltonian (1) as

$$
H(t)=H_{\mathrm{B}}+\sum_{\mu=x, y, z} \sigma_{\mu} f_{\mu}(t) A_{\mu} .
$$

The switching functions ${ }^{1} f_{\mu}(t)$ are the effect of the stroboscopic pulsing of the qubit. They are piecewise constant functions with values \pm 1 :

$$
\begin{gathered}
f_{z}(t)=(-1)^{j} \quad \text { for } \quad t \in\left(t_{j}^{x}, t_{j+1}^{x}\right], \\
f_{y}(t)=(-1)^{k} \quad \text { for } \quad t \in\left(t_{j, k}^{z}, t_{j+1, k}^{x}\right],
\end{gathered}
$$

with $f_{y}(0)=1$ and $f_{y}(\tau)=-1$, and

$$
f_{x}(t)=f_{z}(t) f_{y}(t) .
$$

The evolution operator can be written in terms of the cumulants $\bar{H}^{(n)}$ as

$$
U(\tau, 0)=\exp \left\{-i \tau \sum_{n=1}^{\infty} \bar{H}^{(n)}\right\},
$$

with $\tau \bar{H}^{(n)} \propto \tau^{n}$. The first and the second cumulants are defined [34] as $\tau \bar{H}^{(1)}=\int_{0}^{\tau} H(t) d t$ and $\tau \bar{H}^{(2)}=$ $-\frac{i}{2 \tau} \int_{0}^{\tau} d t_{1} \int_{0}^{t_{1}} d t_{2}\left[H\left(t_{1}\right), H\left(t_{2}\right)\right]$. From Eqs. (A2) it is straightforward to verify that $\int_{0}^{\tau} d t f_{\mu}(t)=0$ for $\mu=x, y$, or $z$ and for $N_{z}=N_{x}=1$. The first cumulant is proportional to the bath Hamiltonian

$$
\tau \bar{H}^{(1)}=\tau H_{\mathrm{B}} .
$$

For the second cumulant one finds

$$
\begin{aligned}
2 i \tau \bar{H}^{(2)}= & \sum_{\mu=x, y, z} \sigma_{\mu} I_{2}^{\mu}\left[H_{\mathrm{B}}, A_{\mu}\right] \\
& +\sum_{\mu, \nu=x, y, z} I_{2}^{\mu, \nu}\left[\sigma_{\mu} A_{\mu}, \sigma_{\nu} A_{\nu}\right],
\end{aligned}
$$

with the integrals

and

$$
I_{2}^{\mu}:=\int_{0}^{\tau} d t_{1} \int_{0}^{t_{1}} d t_{2}\left(f_{\mu}\left(t_{2}\right)-f_{\mu}\left(t_{1}\right)\right)
$$

$$
I_{2}^{\mu, v}:=\int_{0}^{\tau} d t_{1} \int_{0}^{t_{1}} d t_{2} f_{\mu}\left(t_{2}\right) f_{v}\left(t_{1}\right) .
$$

\footnotetext{
${ }^{1}$ The form of the switching function can be easily understood if one remembers that an $X \pi$ pulse changes signs in front of $Y$ and $Z$ coupling, while a $Z \pi$ pulse changes signs in front of $X$ and $Y$ coupling.
} 
The integrals (A7a) and (A7b) can be easily evaluated; we report below only those that are different from zero. For $N_{z}=N_{x}=1$ one finds

$$
I_{2}^{y}=\frac{\tau^{2}}{4}, \quad I_{2}^{z}=\frac{\tau^{2}}{2}, \quad I_{2}^{x, z}=-I_{2}^{z, x}=-\frac{\tau^{2}}{4} .
$$

The second cumulant then becomes

$$
\begin{aligned}
2 i \tau \bar{H}^{(2)}= & \frac{\tau^{2}}{4} \sigma_{y}\left[H_{\mathrm{B}}, A_{y}\right]+\frac{\tau^{2}}{2} \sigma_{z}\left[H_{\mathrm{B}}, A_{z}\right] \\
& +i \frac{\tau^{2}}{2} \sigma_{y}\left[A_{x}, A_{z}\right]_{+},
\end{aligned}
$$

where the notation [ $]_{+}$stands for an anticommutator. It is interesting to notice that $\bar{H}^{(2)}$ does not contain any terms proportional to $\sigma_{x}$.

If the Hamiltonian is $\mathrm{SU}(2)$ invariant and the coupling constants $\hat{J}_{0}^{i j}$ and $\hat{J}_{1}^{i}$ are equal and independent of the indices $i$ and $j\left(\hat{J}_{0}^{i j}=\alpha \lambda\right.$ and $\left.\hat{J}_{1}^{i}=\lambda\right)$, the commutators in Eq. (A9) vanish because the Pauli matrices anticommute. This is true for a central spin model.

On the other hand, if $A_{\mu} \equiv \sigma_{\mu}^{(1)}$ (e.g., for a spin chain), the commutators in Eq. (A9) are different from zero. The anticommutator becomes $\left[A_{x}, A_{z}\right]_{+}=2 i \sum_{i, j}\left(\hat{J}_{1}\right)_{x x}\left(\hat{J}_{1}\right)_{z z}(1-$ $\left.\delta_{i j}\right) \sigma_{x}^{(i)} \sigma_{z}^{(j)}$ which implies that it vanishes for a spin chain because the qubit is coupled to a single site only (i.e., $i=j$ holds).

As usual we are interested in the difference between the evolved density matrix $\rho_{0}^{(\gamma)}(\tau)=U(\tau, 0) \rho_{0}^{(\gamma)} U(\tau, 0)^{\dagger}$ and the initial one. We find

$$
\begin{aligned}
\operatorname{Tr}_{\mathrm{B}}\left\{\rho_{0}^{(\gamma)}(\tau)-\rho_{0}^{(\gamma)}\right\}= & \frac{\tau^{2}}{4}\left[\sigma_{y}, \rho_{\mathrm{S}}^{(\gamma)}\right] \operatorname{Tr}_{\mathrm{B}}\left\{\rho_{\mathrm{B}}\left[A_{x}, A_{z}\right]_{+}\right\} \\
& +\frac{\tau^{4}}{16} c_{y y}^{(\gamma)} \operatorname{Tr}_{\mathrm{B}}\left\{\left[A_{x}, A_{z}\right]_{+} \rho_{\mathrm{B}}\left[A_{x}, A_{z}\right]_{+}\right\},
\end{aligned}
$$

where the hermiticity $A_{\mu}^{\dagger}=A_{\mu}$ was used. In writing Eq. (A10) we neglected the contributions coming from the first cumulant because they do not alter the qubit-operator content of the norm distance.

If $\rho_{\mathrm{B}} \propto \mathbb{1}_{\mathrm{B}}$ the terms with the trace over the bath vanish for an SU(2)-invariant Hamiltonian (see Sec. III) while they are finite for an asymmetric model such as the one in Eq. (1) in Sec. II.
In order to understand why the distance norm scales with exponents $N_{z}+1$ for $N_{x}=1$ (and in general for $N_{x}$ odd) in Table IV(a), some knowledge of the third cumulant $\bar{H}^{(3)}$ is required. This cumulant is defined [34] as

$$
\begin{aligned}
-6 \tau \bar{H}^{(3)}= & \int_{0}^{\tau} d t_{1} \int_{0}^{t_{1}} d t_{2} \int_{0}^{t_{2}} d t_{3}\left\{\left[H\left(t_{3}\right),\left[H\left(t_{2}\right), H\left(t_{1}\right)\right]\right]\right. \\
& \left.+\left[H\left(t_{1}\right),\left[H\left(t_{2}\right), H\left(t_{3}\right)\right]\right]\right\} .
\end{aligned}
$$

The commutators $\left[H\left(t_{2}\right), H\left(t_{1}\right)\right]$ and $\left[H\left(t_{2}\right), H\left(t_{3}\right)\right]$ have the same operator content as $\bar{H}^{(2)}$, but differ in their prefactors and in their time dependence. One can verify that $\left[H_{\mathrm{B}}, \bar{H}^{(2)}\right]=$ 0 if the bath Hamiltonian is $\mathrm{SU}(2)$ invariant due to the anticommutation of the Pauli matrices. For the same reason one finds that

$$
\left[\sum_{\mu} \sigma_{\mu} A_{\mu}, \bar{H}^{(2)}\right] \propto \sum_{\mu, \nu} \epsilon_{\mu, y, \nu} \sigma_{\nu} \otimes \sigma_{x}^{(i)} \sigma_{y}^{(j)} \sigma_{\mu}^{(k)},
$$

where $i \neq j \neq k$. Equation (A12) only provides the operator contents of Eq. (A11). In order to eliminate the time dependence one must substitute Eq. (A12) into Eq. (A11) and integrate over $t_{1}, t_{2}$, and $t_{3}$. Each operator $A_{\mu}$ brings a switching function $f_{\mu}(t)$ with it, such that the integration in (A11) yields the coefficients

$$
I_{3}^{\alpha, \beta, \gamma}=\int_{0}^{\tau} d t_{1} \int_{0}^{t_{1}} d t_{2} \int_{0}^{t_{2}} d t_{3} f_{\alpha}\left(t_{1}\right) f_{\beta}\left(t_{2}\right) f_{\gamma}\left(t_{3}\right) .
$$

The indeces $\alpha, \beta$, and $\gamma$ can be equal to $x, y$, and $z$. We have checked numerically that for $N_{x}=1$ and $N_{z}=2$ the only nonzero contributions are given by $I_{3}^{x, z, z}, I_{3}^{z, x, z}$, and $I_{3}^{z, z, x}$ corresponding to the qubit operator $\sigma_{x}$. Thus the third cumulant $\bar{H}^{(3)}$ is a term of pure dephasing that can be suppressed by means of a $Z$ sequence of $\pi$ pulses. From the numerical results we expect that the same argument holds in general for higher cumulants and for $N_{x}$ odd.

We also checked our results for a spin chain. We find the same results as for a central spin model in the cases of low symmetry, high symmetry, and in the case of an asymmetric Hamiltonian with $\rho_{\mathrm{B}} \propto \mathbb{1}_{\mathrm{B}}$. Discrepancies are found for the $\mathrm{SU}(2)$-invariant Hamiltonian in combination with the product state $\left|\psi_{\mathrm{B}}\right\rangle$ (5). A possible explanation is provided by the commutators in Eq. (A9) that do not vanish for a spin chain with $A_{\mu}=\sigma_{\mu}^{(1)}$. Hence the precise topology of the model matters for the case of mixed degree of symmetry.
[1] L. Viola and S. Lloyd, Phys. Rev. A 58, 2733 (1998).

[2] E. L. Hahn, Phys. Rev. 80, 580 (1950).

[3] H. Y. Carr and E. M. Purcell, Phys. Rev. 94, 630 (1954).

[4] S. Meiboom and D. Gill, Rev. Sci. Instrum. 29, 688 (1958).

[5] G. S. Uhrig, Phys. Rev. Lett. 98, 100504 (2007).

[6] W. Yang and R.-B. Liu, Phys. Rev. Lett. 101, 180403 (2008).

[7] G. S. Uhrig, New J. Phys. 10, 083024 (2008).

[8] G. S. Uhrig, Phys. Rev. Lett. 102, 120502 (2009).

[9] M. J. Biercuk, H. Uys, A. P. VanDevender, N. Shiga, W. M. Itano, and J. J. Bollinger, Nature (London) 458, 996 (2009).
[10] H. Uys, M. J. Biercuk, and J. J. Bollinger, Phys. Rev. Lett. 103, 040501 (2009).

[11] K. Khodjasteh, T. Erdélyi, and L. Viola, Phys. Rev. A 83, 020305 (2011).

[12] K. Khodjasteh and D. A. Lidar, Phys. Rev. Lett. 95, 180501 (2005).

[13] J. R. West, B. H. Fong, and D. A. Lidar, Phys. Rev. Lett. 104, 130501 (2010).

[14] S. Pasini and G. S. Uhrig, J. Phys. A 41, 312005 (2008).

[15] Z.-Y. Wang and R.-B. Liu, Phys. Rev. A 83, 022306 (2011).

[16] L. Jiang and A. Imambekov, e-print arXiv:1104.5021. 
[17] W.-J-Kuo and D. A. Lidar, e-print arXiv:1106.2151.

[18] G. Quiroz and D. A. Lidar, e-print arXiv:1105.4303.

[19] L. Cywiński, R. M. Lutchyn, C. P. Nave, and S. Das Sarma, Phys. Rev. B 77, 174509 (2008).

[20] S. Pasini and G. S. Uhrig, Phys. Rev. A 81, 012309 (2010).

[21] J. Du, X. Rong, N. Zhao, Y. Wang, J. Yang, and R. B. Liu, Nature (London) 461, 1265 (2009).

[22] A. Ajoy, G. A. Álvarez, and D. Suter, Phys. Rev. A 83, 032303 (2011).

[23] E. R. Jenista, A. M. Stokes, R. T. Branca, and W. S. Warren, J. Chem. Phys. 131, 204510 (2009).

[24] J. Schliemann, A. Khaetskii, and D. Loss, J. Phys. Condens. Matter 15, R1809 (2003).

[25] M. Bortz and J. Stolze, J. Stat. Mech. (2007) P06018.

[26] M. Bortz and J. Stolze, Phys. Rev. B 76, 014304 (2007).
[27] W. M. Witzel and S. Das Sarma, Phys. Rev. B 77, 165319 (2008).

[28] B. Lee, W. M. Witzel, and S. Das Sarma, Phys. Rev. Lett. 100, 160505 (2008).

[29] S. Pasini, P. Karbach, and G. S. Uhrig, Europhys. Lett. 96, 10003 (2011).

[30] D. A. Lidar, P. Zanardi, and K. Khodjasteh, Phys. Rev. A 78, 012308 (2008).

[31] G. S. Uhrig and D. A. Lidar, Phys. Rev. A 82, 012301 (2010).

[32] G. A. Álvarez, A. Ajoy, X. Peng, and D. Suter, Phys. Rev. A 82, 042306 (2010).

[33] D. Li, Y. Dong, R. G. Ramos, J. D. Murray, K. MacLean, A. E. Dementyev, and S. E. Barrett, Phys. Rev. B 77, 214306 (2008).

[34] U. Haeberlen, High Resolution NMR in Solids: Selective Averaging (Academic Press, New York, 1976).

[35] W. Magnus, Commun. Pure Appl. Math. 7, 649 (1954). 